\title{
Radiative Heat and Mass Transfer of an MHD Free Convection Flow Along a Stretching Sheet with Chemical Reaction, Heat Generation and Viscous Dissipation
}

\author{
Ishrat Zahan and M. A. Samad \\ Department of Mathematics, University of Dhaka, Dhaka-1000, Bangladesh \\ Received on 17.08.2011. Accepted for Publication on 18. 09.2012
}

\begin{abstract}
In the present study, an analysis is carried out to investigate the effect of chemical reaction and radiation on a steady two-dimensional magneto-hydrodynamics (MHD) heat and mass transfer free convection flow of a viscous incompressible fluid along a stretching sheet with heat generation along with the effect of viscous dissipation. The basic non-linear partial differential equations governing the flow field are reduced to a system of coupled non-linear ordinary differential equations by similarity transformations and the equations are solved numerically by applying Nachtsheim-Swigert shooting iteration technique along with sixth order Runge-Kutta integration scheme. The numerical results with respect to embedded parameters are displayed graphically for the non-dimensional velocity, temperature and concentration profiles. Finally the effects of the pertinent parameters which are of physical and engineering interest are presented in tabular form.
\end{abstract}

Keywords: Boundary layer, buoyancy parameter, Eckert number, heat transfer, magnetic field parameter, radiation parameter, viscous dissipation.

\section{Introduction}

Incompressible viscous flow involving heat and mass transfer under the influence of chemical reaction and radiation is of great importance to many areas of science and engineering. This is because of various possible engineering and metallurgical applications such as hot rolling, wire drawing, metal and plastic extrusion, continuous casting, glass fiber product, crystal growing, paper product, petrochemical industry, power and cooling systems, cooling of nuclear reactors, heat exchange design, dynamics as well as in magneto-hydrodynamics power generation systems. Many analytical and numerical studies have been conducted to explain the various aspects of boundary layer flow with heat and mass transfer over flat surface.

Sakiadis $[1,2]$ was the first to study boundary layer flow over a stretching surface moving with a constant velocity in an ambient fluid. He employed a similarity transformation and obtained a numerical solution for the problem. Erickson et al. [3] extended the work of Sakiadis to account for mass transfer at the stretching surface. Tsou et al. [4] presented a combined analytical and experimental study of the flow and temperature field in the boundary layer on a continuous moving surface, in which the flow is caused by an elastic sheet moving in its own plane with a velocity varying linearly with the distance from a fixed point studied by Crane [5].

Possible heat generation effect may alter the temperature distribution; consequently, the particle deposition rate in nuclear reactors, electronic chips and semi conduction wafers. Vajravelu and Hahjinicalaous [6] studied the heat transfer characteristic in the laminar boundary layer of a viscous fluid over a stretching surface with viscous dissipation in the presence of internal heat generation or absorption. Gebhart [7] was the first who studies the problem taking into account the viscous dissipation. Recently, Samad and Tania investigated the effect of radiation, heat generation and viscous dissipation on MHD free convection flow along a stretching sheet [8].

Diffusion of a chemically reactive space from a stretching sheet was studied by Anderson et al. [9]. Anjali Devi and Kandasamy [10] have analyzed the effects of chemical reaction, heat and mass transfer on laminar flow along a semi infinite flow plate. In the present work, we make an attempt to investigate the problem of MHD free convection flow past an infinite or semi-infinite vertical stretching sheet with chemical reaction, radiation, and heat generation. In this work an attempt is also taken to focus the effect of viscuss dissipation, To be more specific, we have studied and investigated the presence of radiation effect on MHD free convection heat and mass transfer flow along a stretching sheet as its importance in engineering and industrial applications.

\section{Mathematical Formulation}

Let us consider a steady two dimensional MHD free convection laminar boundary layer flow of an electrically conducting fluid over a continuous stretching vertical sheet in the presence of heat generation under the influence of thermal radiation. Here the system is taken to be Cartesian coordinate system with $\mathrm{x}$-axis and $\mathrm{y}$-axis in the vertical and horizontal direction respectively. The temperature and the concentration of the free stream are $T_{w}$ and $C_{W}$ whereas the ambient temperature and the concentration of the free stream are $T_{s}$ and $C_{s \mathrm{~s}}$.

The fluid is taken to be gray, absorbing or emitting radiation but being a nonscattering medium. To describe the radioactive heat flux in the energy equation the Rosseland approximation is used. The radioactive heat flux in the $\mathrm{x}$-direction is negligible in comparison to the $\mathrm{y}$-direction. The effect of viscous dissipation is taken into accounted and the concentration is assumed to be non-reactive. To formulate the problem the two dimensional continuity equation is introduced and on the momentum boundary layer equation the left hand side consists of the acceleration terms 
and the right hand side consists of several terms in which the first term indicates the viscous term, the second term indicates the buoyancy terms and the third term indicates the presence of magnetic field. On the energy equation a new term corresponding to radiation and a viscous term with the other usual terms is introduced and the concentration equation consists of the usual terms.

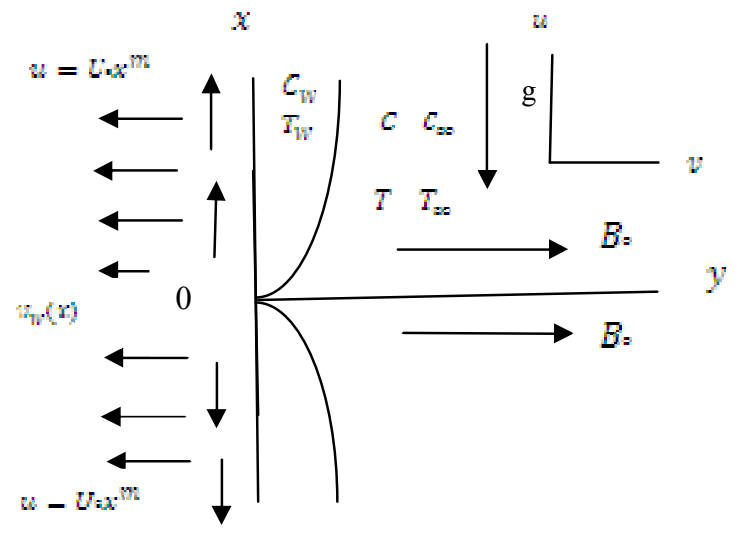

Fig. 1. Sketch of the physical model

Equation of Continuity:

$$
\frac{\partial u}{\partial x}+\frac{\partial v}{\partial y}=0
$$

Momentum equation:

$$
\begin{aligned}
& u \frac{\partial u}{\partial x}+v \frac{\partial u}{\partial y}=v \frac{\partial^{2} u}{\partial y^{2}}+\operatorname{sg} \beta\left(T-T_{\infty}\right)- \\
& \frac{\sigma D^{2}}{\rho} u
\end{aligned}
$$

Energy equation:

$$
\begin{aligned}
& u \frac{\partial T}{\partial x}+\nu \frac{\partial T}{\partial y}=\frac{\kappa}{\rho C_{p}} \frac{\partial^{2} T}{\partial y^{2}}+\frac{Q_{p}}{\rho C_{\gamma}}\left(T-T_{s}\right)- \\
& \frac{1}{\rho C_{\nu}} \frac{\partial q_{\gamma}}{\partial y}+\frac{\nu}{C_{\nu}}\left(\frac{\partial u}{\partial y}\right)^{2}
\end{aligned}
$$

Concentration Equations:

$u \frac{\partial c}{\partial x}+v \frac{\partial c}{\partial y}=D_{m} \frac{\partial^{2} c}{\partial y^{2}}$

Boundary conditions are:

$$
\begin{aligned}
& u=U \cdot x^{m}, v=v_{w}, T=T_{w}, C=C_{w} \text { at } y=0 \\
& u=0, I^{n}-Y_{\infty}=Y_{w}^{*}-T_{\infty}=a x^{n}, C^{n}-C_{\infty} \\
& =C_{w}-C_{\infty}=b x^{2} \text { as } y \rightarrow \infty
\end{aligned}
$$

Where $\mathrm{u}$ and $\mathrm{v}$ are the velocity components in the $\mathrm{x}$ and $\mathrm{y}$ direction respectively, $v$ is the kinematic viscosity, $\mathrm{g}$ is the acceleration due to gravity, $\beta$ is the volumetric coefficient of thermal expansion, $T$ is the fluid temperature in the boundary layer, $\mathrm{C}$ is the concentration of the fluid with in boundary layer, $\sigma$ is the electric conductivity, $B$ : is the uniform magnetic field strength, $\rho$ is the density of the fluid, $k$ is the thermal conductivity of the fluid, $C_{p}$ is the specific heat at constant pressure, $Q=$ is the heat generation constant, $q_{r}$ is the radiative heat flux and $D_{m}$ is the coefficient of mass diffusivity.

The effect of the second term on the right hand side of the momentum equation is due to buoyancy force. If $s=0$, there is no buoyancy effect on the flow and the governing equations represent forced convection flow. In this work we are considering the case $\mathrm{s}=1.0$ i.e. the free convection flow where the buoyancy effect is dominant. In order to obtain a solution of equations (1)-(4), we introduce the following similarity variables

$$
\begin{aligned}
& \eta=y \sqrt{\frac{m+1}{2}} \sqrt{\frac{U x^{m-1}}{v}} \\
& \psi=\sqrt{\frac{2 v}{m+1}} \sqrt{U x^{m+1}} f(\eta) \\
& I-I_{\infty}=a x^{n} \theta(\eta) \\
& C^{\prime}-C_{\infty}=b x^{2} \varphi(\eta)
\end{aligned}
$$

Where $\psi$ is the stream function, $\eta$ is dimensionless distance normal to the sheet, $\mathrm{f}$ is the dimensionless stream function, $\theta$ is the dimensionless fluid temperature and $\psi$ is the dimensionless concentration.

Now,

$$
\begin{aligned}
& u=U_{s} x^{m} f^{\prime}(\eta) \\
& v=\sqrt{\frac{2 v U:}{m+1} x^{\frac{m-1}{2}}\left[\frac{m+1}{2} \rho(\eta)+\eta \frac{m-1}{2} f^{\prime}(\eta)\right]}
\end{aligned}
$$

Substituting in the governing equations gives rise to the following boundary value problem

$$
\begin{aligned}
& f^{m} \text { । } f f^{\prime \prime} \quad \begin{array}{c}
2 m \\
m+1
\end{array} f^{2} \text { । } \begin{array}{c}
2 \lambda \\
m+1
\end{array} \theta \\
& -\frac{2 M}{6 m+1)} f^{\prime}=0 \\
& \theta^{\prime \prime} \text { । । }{ }_{3 N+4}^{3 N P r} f \theta^{\prime} \mid \underset{(m+1)(3 N+4)}{6 N P_{r}}(Q \\
& \left.n f^{\prime}\right) \theta+\frac{3 N}{3 N+4} \operatorname{Pr} E C f^{\prime \prime 2}=0 \\
& \varphi^{\prime \prime}+5 c \varphi^{\prime} f-\frac{2 l}{m+1} S c \varphi f^{\prime}=0
\end{aligned}
$$

$M=\frac{2 \sigma^{2} \mathrm{w}^{1-m}}{\rho \mathrm{i}}$ is the local magnetic parameter.

$\lambda=\frac{G r_{x}}{R e_{x}^{2}}=\frac{g \beta a x^{n-2 m+1}}{U_{i}^{n}}$

is the local buoyancy parameter. 
$E c=\frac{\left(U w^{m}\right)^{2}}{C_{p}\left(T-T_{w}\right)}$ is the Eckert number.

$\mathrm{Pr}^{\mathrm{r}}=\frac{\mu C_{p}}{\kappa}$ is the Prandtl number

$N=\frac{\kappa \kappa_{1}}{4 \sigma_{1} T_{\infty}^{3}}$ is the radiation parameter.

$Q=\frac{Q:}{\rho C_{\gamma} V_{x} x^{m-1}}$ is the local heat source parameter.

$S c=\frac{v}{D_{n}}$ is the Schmidt number.

and the boundary conditions becomes

$f^{\prime}=1, f=F w, \theta=1, \varphi=1$ at $\eta=0$

$f^{\prime}=0, \quad \theta=0, \quad \varphi=0$ as $\eta \rightarrow \infty$

$F w=-v_{w} \sqrt{\frac{2}{m+1}} \sqrt{\frac{x^{2-m}}{w U:}}$ is the suction parameter.

\section{Numerical Computation}

By applying a shooting method with Nachtsheim-swigert iteration technique [11] along with the sixth order Runge-kutta Butcher iteration scheme the numerical solution of the non-linear differential equations (6) - (8) under the boundary conditions (9) have been performed. Here in all cases the step size $\Delta \eta=0.01$ is chosen to satisfy the convergence criterion of $10^{-6}$. The value of $\eta_{\infty}=\eta_{\infty}+\Delta \eta$. The maximum value of $\eta_{\infty}$ to each group of parameters $\lambda$, Pr, $F w, M, S c, Q, N, E c, m, n$ and $l$ is determined when the value of the unknown boundary conditions at $\eta=0.0$ change to successful loop with error less than $10^{-6}$. In order to verify the effects of the step size $\Delta \eta$, the programming code was run for our model with three different step sizes as $\Delta \eta=0.005, \Delta \eta=0.007, \Delta \eta=0.01$ and in each case we found excellent agreement among them.

The parameter of engineering interest of the present problem are the Skin friction coefficient $\left(C_{f}\right)$, Nusselt number $(\mathrm{N} u)$ and Sherwood number ( $5 h$ ) which represent physically wall shear stress, local wall heat transfer rate and wall mass transfer rate respectively.

From equation (5) we write,

$$
\frac{\partial u}{\partial v}=U x^{\frac{m-1}{2}} \sqrt{\frac{(m+1) v}{2 v}} f^{\prime \prime}(\eta)
$$

Wall shear stress is given by

$\tau_{w}=\left.\mu \frac{\partial u}{\partial y}\right|_{y=0}=\mu U \cdot x^{\frac{3 m-1}{2}} \sqrt{\frac{(m+1) U}{2 u}} f^{\prime \prime}(0)$

Hence the skin friction coefficient is given as, $C_{f}=\frac{\tau_{W}}{\frac{\pi}{2} p U^{2}}=\sqrt{\frac{2(m+1)}{R s_{w}}} f^{\prime \prime}(0)$

$R e_{x}-\frac{U: x^{m+1}}{v}$ is the local Reynolds number.

The local heat flux $q_{r}$ can be written by Using Fourier's low

$$
\begin{aligned}
& q_{n}=\left.\kappa \frac{\partial T}{a_{y}}\right|_{y=0}= \\
& -\kappa a x^{\%} \sqrt{\frac{m+1}{2}} \sqrt{\frac{U \cdot x^{m-1}}{v}} \theta^{\prime}(0) \\
& \text { Hence the local Nusselt number }(N u) \text { is as, } \\
& N u=\frac{x q_{w}}{\kappa\left(T-T_{\infty}\right)}=-\sqrt{\frac{m+1}{2}} \sqrt{R e_{x}} \theta^{\prime}(0)
\end{aligned}
$$

Now the mass flux $\left(M_{w}\right)$ at the wall is given by

$$
\begin{aligned}
M_{w} & =-\left.D_{m}\left(\frac{\partial c}{\partial y}\right)\right|_{y=0} \\
& =-D_{m} b x^{2} \sqrt{\frac{m+1}{2}} \sqrt{\frac{U x^{m-1}}{v}} \varphi^{\prime}(0)
\end{aligned}
$$

So the local Sherwood number $(S h)$ is given by $S h=\frac{x M_{w}}{I_{\mathrm{n} z}\left(c_{w}-c_{w}\right)}=-\sqrt{\frac{m+1}{2}} \sqrt{F e_{x}} \phi^{\prime}(0)$ Thus from the above equations it can be seen that the skin friction coefficient, Nusselt number and Sherwood number are proportional to $f^{\prime \prime}(0),-\theta^{\prime}(0)$ and $-\varphi^{\prime}(0)$ respectively.

\section{Result and Discussion}

For the purpose of discussing the results, the numerical calculations are presented in the form of non-dimensional velocity, temperature and concentration profiles for different values of buoyancy parameter $(\lambda)$, suction parameter $(F w)$, Prandtl number $(\operatorname{Pr})$, local magnetic parameter $(M)$, heat source parameter $(Q)$, Schmidt number $(S c)$ and local radiation parameter $(N)$. The effects of buoyancy parameter $(\lambda)$ on the velocity, temperature and concentration profiles are shown in Fig 2(a), Fig 2(b) and Fig 2(c) respectively. In Fig 2(a) we observe that the velocity profiles increase with the increase of buoyancy parameter (2) up to around $\eta \Xi 3.8$ but after that the velocity profiles shows a slight decreasing trend as $\eta$ increases further; thus we get a cross flow here. However, near the surface the velocity profiles act quit differently for different values of $\lambda$. The velocity profiles decrease for $\lambda=1.0,2.0$, $3.0,5.0$, whereas the velocity profile increases quite rapidly for $\lambda=7.0$ near the stretching sheet and then starts to decrease. Fig 2(b) and Fig 2(c) exhibit that the temperature and the concentration profiles are strictly decreasing with the increase of buoyancy parameter $\lambda$. In Fig 3(a), Fig 3 (b) and Fig 3(c), we observe that the dimensionless velocity, temperature and concentration profiles decrease with the increase of suction parameter $(F w)$. For $F w=1.0$,we notice that the velocity and temperature profiles increase near the surface and then start to decrease. The velocity profiles decrease with the increase of suction parameter $F w$, expressing the fact that suction stabilized the boundary layer growth. However, for the value of the suction parameter, the temperature and concentration profiles starts to decrease monotonically from the very beginning. So suction can be used very effectively in controlling the flow field. 


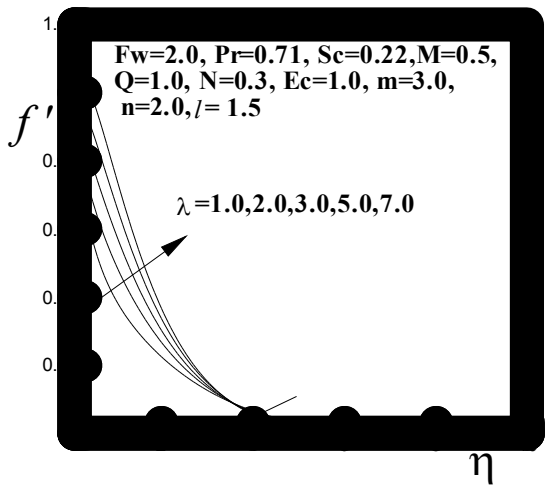

Fig. 2(a). Buoyancy parameter effect on velocity profiles

The effect of Prandtl number (Pr) on velocity, temperature and concentration distribution is displayed in Fig 4(a), Fig 4(b) and Fig 4(c) respectively.

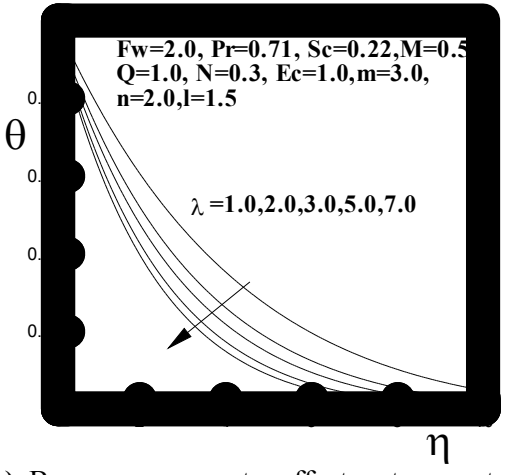

Fig. 2(b). Buovancy parameter effect on temperature profiles

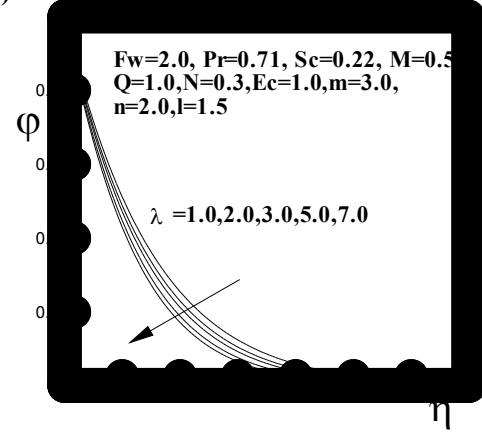

Fig. 2(c).Buoyancy parameter effect on Concentration profiles

Fig 4(a) and Fig 4(b) shows that the velocity and temperature profiles decrease with the increase of Prandtl number (Pr). Physically, $\mathrm{Pr}=0.71,1.0,7.0$, correspond to air at $20^{\circ} \mathrm{c}$, to electrolyte solution such as salt water and to water respectively. We can observe the effect of magnetic parameter $(M)$ on the dimensionless velocity, temperature and concentration distribution from Fig 5. The velocity curve in Fig 5(a) shows that the rate of transport is considerably reduced with the increase of magnetic field parameter $(M)$. It clearly indicates that the transverse magnetic field opposes the transport phenomena. This is due to the fact that the variation of $M$ leads to the variation of Lorenz force due to magnetic field and the Lorenz force produces more resistance to the transport phenomena. However, the temperature and concentration profiles increase as magnetic parameter $(M)$ increase.The profile of velocity, temperature and concentration for different values of heat source parameter $(Q)$ are shown in Fig 6. It is observed that as $(Q)$ increase

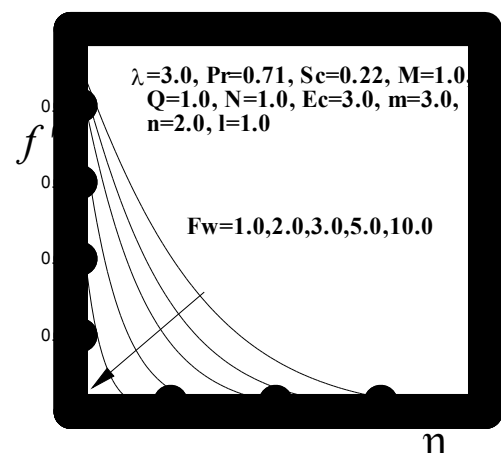

Fig. 3(a). Suction parameter effect on velocity profiles

the velocity and temperature increase rapidly and overshoot. But (Q) increase furthers the rate decrease and at $Q=4.0$ (around) the temperature gradient is zero and the sheet starts to transfer heat to environment.

For $Q=3.0$ there exists overshooting in the temperature profiles. From Fig 7(a) and Fig 7(b)

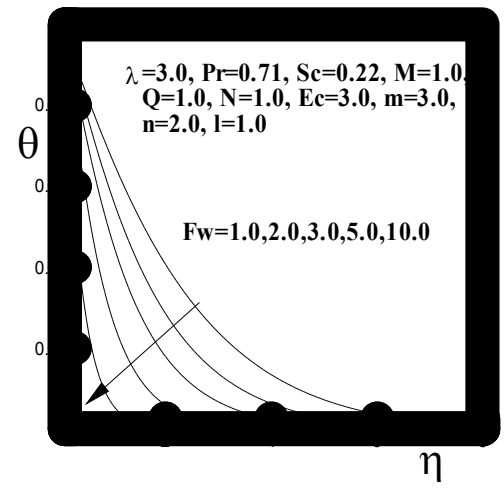

Fig. 3(b). Suction parameter effect on temperature profiles

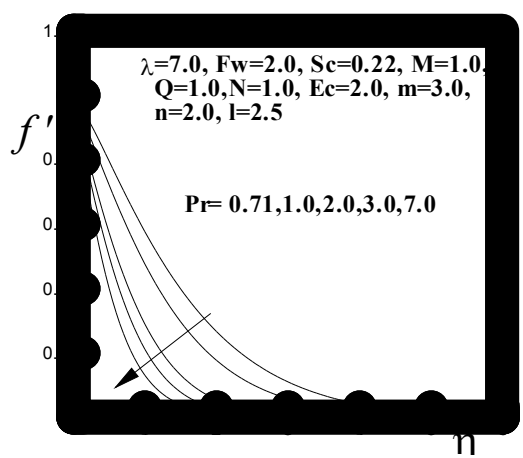

Fig. 3(c). Suction parameter effect on concentration profiles 


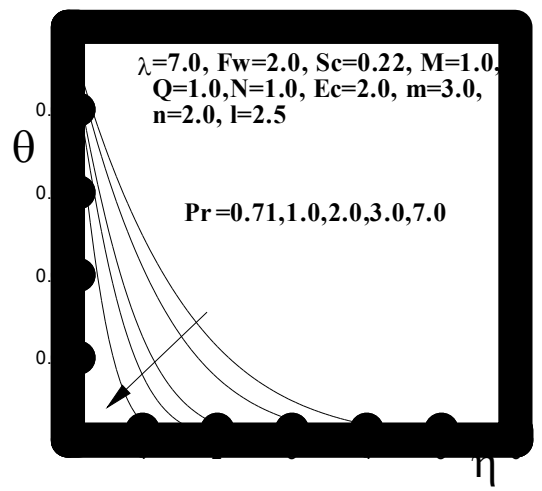

Fig. 4(a). Prandtl number effect on velocity profiles

we see that for $S c=0.22,0,4,0,6,1,0$, there is almost no variation in the corresponding velocity profiles. However the concentration profiles show significant variation for different values of Schmidt number $(50)$.

In Fig 8 the velocity and temperature decrease with the increase of radiation parameter $(N)$, whereas the concentration profiles increase. The velocity profiles rise near the surface for $N \leq 0.5$. So radiation can be used to control velocity and temperature boundary layers quite effectively.

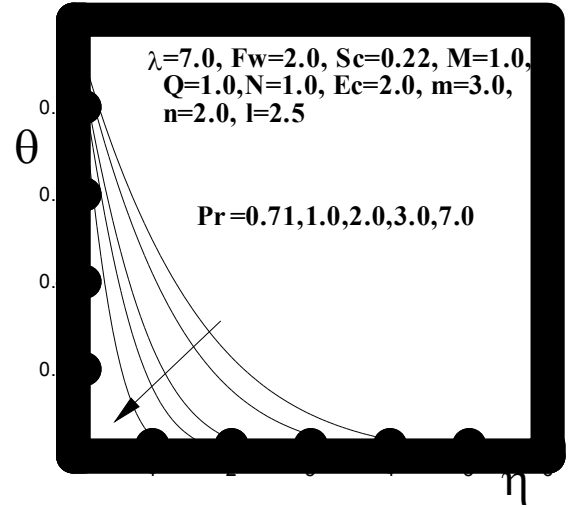

Fig. 4(b). Prandtl number effect on temperature profiles

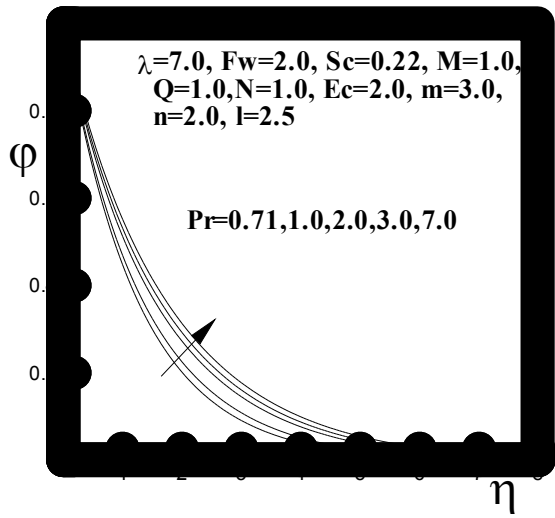

Fig. 4(c). Prandtl number effect on concentration profiles

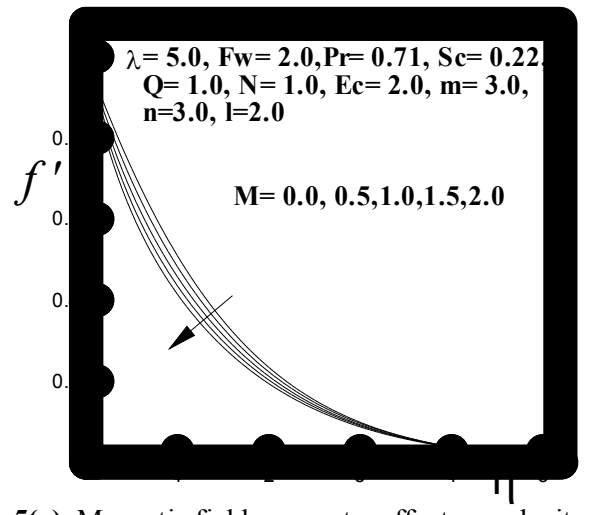

Fig. 5(a). Magnetic field parameter effect on velocity profiles

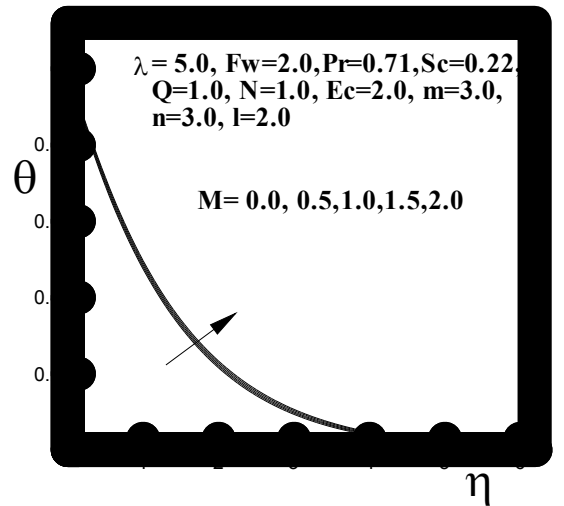

Fig. 5(b). Magnetic field parameter effect on temperature profiles

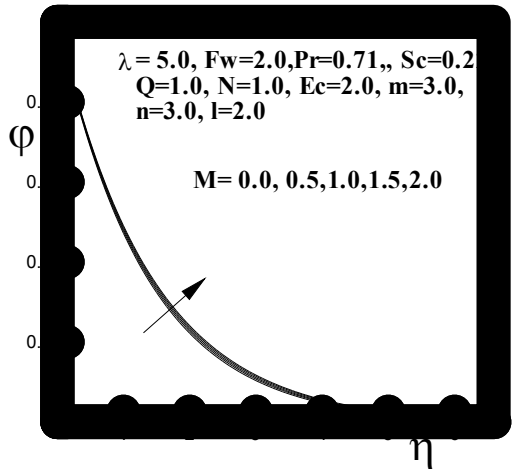

Fig. 5(c). Magnetic field parameter effect on concentration profiles

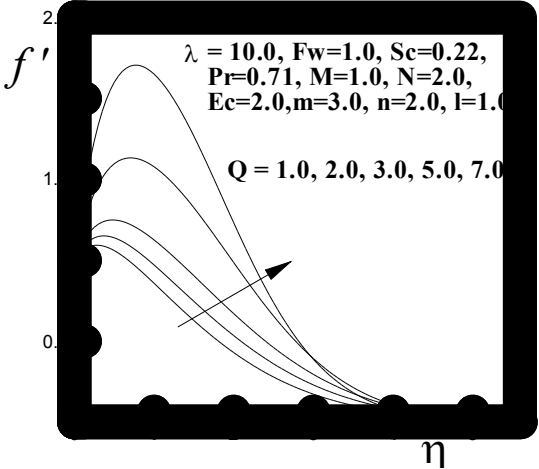

Fig. 6(a). Heat source parameter effect on velocity profiles 


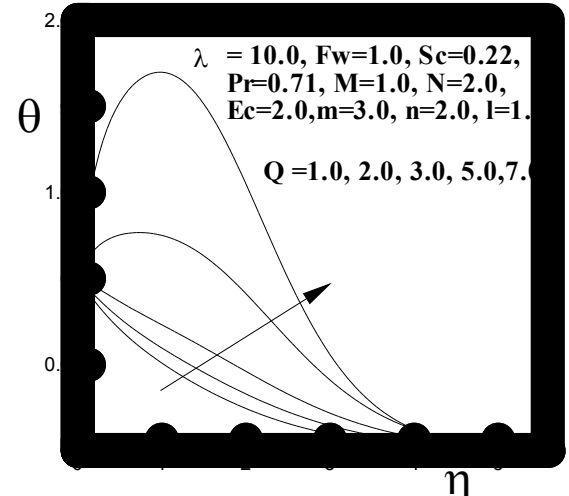

Fig. 6(b). Heat source parameter effect on temperature profiles

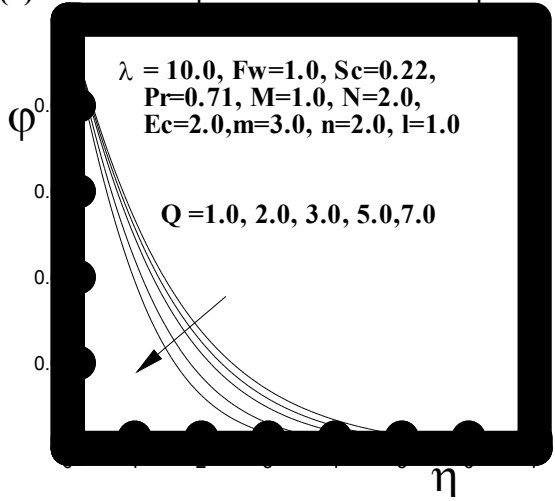

Fig. 6(c). Heat source parameter effect on concentration profiles

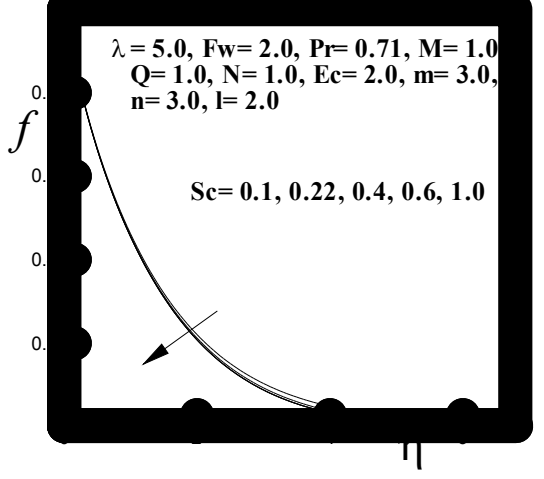

Fig. 7(a). Schmidt number effect on velocity profiles

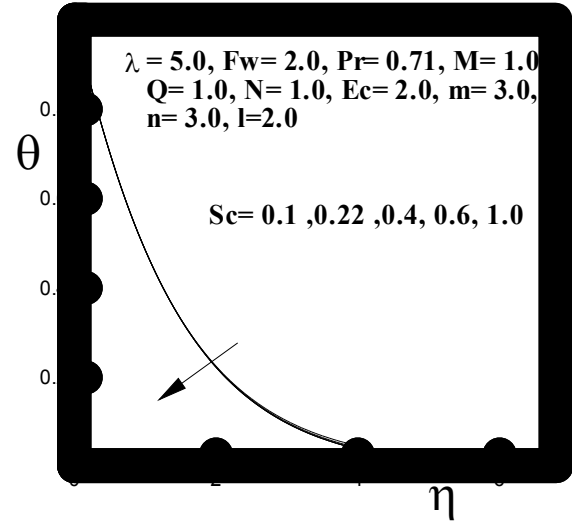

Fig. 7(b). Schmidt number effect on temperature profiles

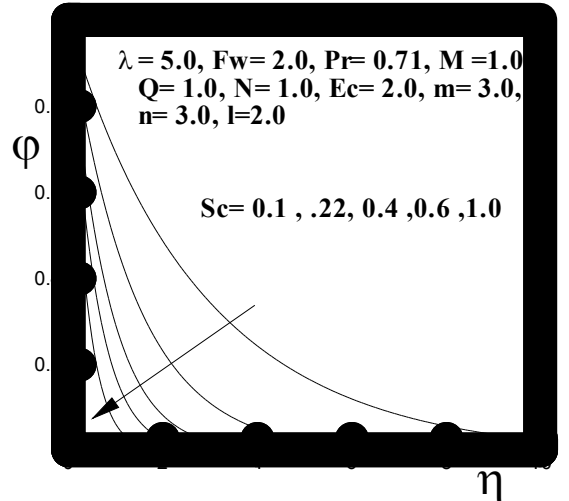

Fig. 7(c). Schmidt number effect on concentration profiles

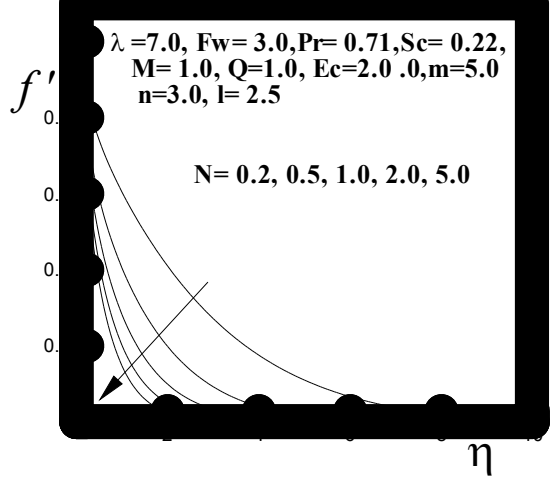

Fig. 8(a). Radiation parameter effect on velocity profiles

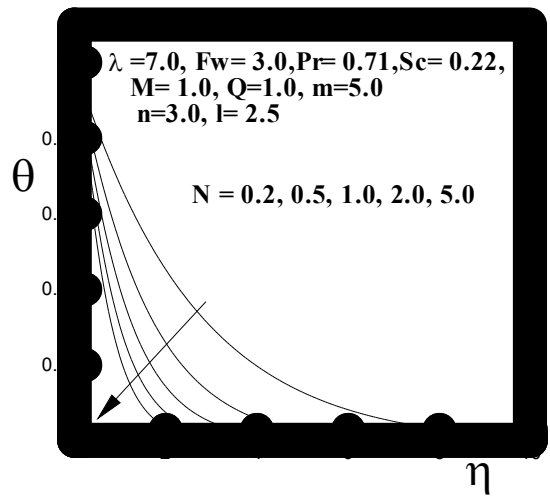

Fig. 8(b).Radiation parameter effect on temperature profiles

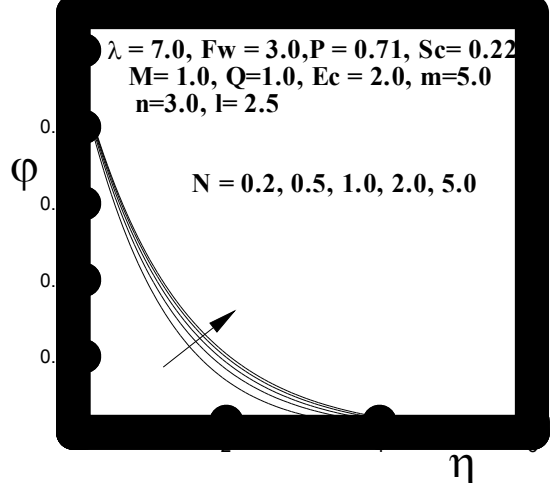

Fig. 8(c). Radiation parameter effect on concentration profiles 
Some effects of the above mentioned parameters like $\operatorname{Pr}, M$, and $\lambda$ on the skin friction coefficient $\left(C_{\gamma}\right)$, Nusselt number $(\mathrm{Nu})$ and Sherwood number $(\mathrm{Sh})$ are shown in the Tables, which demonstrate the comparison of the data produced by the present code and those of M.A. Samad and M. Mohebujjaman [12] (2009). In fact, the results show a close agreement, and hence justify the use of the present code.

Table. 1. Numerical values proportional to $C_{i}, N u$ and $S h$ for different values of $\mathbf{P r}$

\begin{tabular}{|c|c|c|c|c|c|c|}
\hline $\operatorname{Pr}$ & $C_{f}$ & Published & $N u$ & Published & Sh & Published \\
\hline 0.71 & -0.8202594 & -1.0586135 & -0.5029761 & -0.3988216 & 0.4514297 & 0.4070962 \\
\hline 1.0 & -0.9171489 & -1.1387943 & -0.4691028 & -0.3531551 & 0.4336939 & 0.3945218 \\
\hline 2.0 & -1.1243664 & -1.2533078 & -0.3207594 & -0.2852384 & 0.39833880 & 0.3805534 \\
\hline 3.0 & -1.2074031 & -1.3011301 & -0.2554426 & -0.2306239 & 0.3868395 & 0.3761920 \\
\hline 7.0 & -1.3238097 & -1.3671478 & -0.0826424 & -0.0218965 & 0.3751262 & 0.3714634 \\
\hline
\end{tabular}

Table. 2. Numerical values proportional to $C_{i}, N u$ and $5 h$ for different values of $M$

\begin{tabular}{|c|c|c|c|c|c|c|}
\hline$M$ & $C_{f}$ & Published & $N u$ & Published & Sh & Published \\
\hline 0.0 & 0.0239363 & 0.0088494 & -0.4598602 & -0.7831341 & 0.8565031 & 0.7149090 \\
\hline 0.5 & -0.2233846 & -0.1914180 & -0.6523555 & -1.0886432 & 0.8356817 & 0.6999995 \\
\hline 1.0 & -0.4402355 & -0.3692765 & -0.8596322 & -1.4137521 & 0.8180179 & 0.6878163 \\
\hline 1.5 & -0.6330604 & -0.5304114 & -1.0789458 & -1.7513681 & 0.8030096 & 0.6775278 \\
\hline 2.0 & -0.8081618 & -0.6795450 & -1.3039416 & -2.0982242 & 0.7898960 & 0.6686882 \\
\hline
\end{tabular}

Table. 3. Numerical values proportional to $C_{f}, N w$ and $S h$ for different values of $\lambda$

\begin{tabular}{|c|c|c|c|c|c|c|}
\hline$\lambda$ & $C_{f}$ & Published & Nu & Published & Sh & Published \\
\hline 1.00 & -0.2813563 & -0.5832767 & -1.8647538 & -2.2008276 & 1.0289492 & 0.9807700 \\
\hline 3.00 & 0.7212181 & 0.2404924 & $-0.4100951-$ & -0.3366579 & 1.0730380 & 1.0132378 \\
\hline 5.00 & 1.6597346 & 1.0295899 & 0.0984587 & 0.0605359 & 1.1104353 & 1.0430976 \\
\hline 7.00 & 2.5448099 & 1.778432 & 0.0494679 & 0.2515683 & 1.1426757 & 1.0695051 \\
\hline 10.0 & 3.7893009 & 2.8371519 & 0.1780736 & 0.4195393 & 1.1836479 & 1.1036445 \\
\hline
\end{tabular}

Graphical representation of the present and published data for different values of Pr:

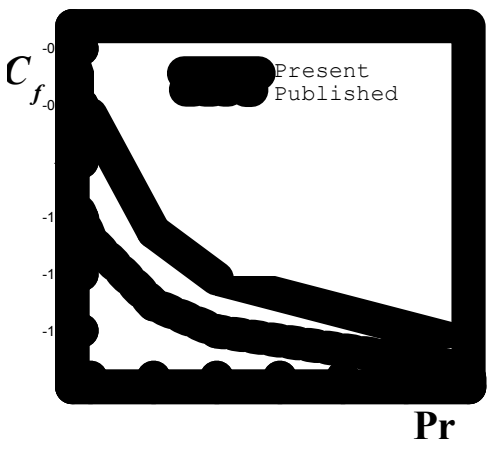

\section{Conclusion}

By summarizing the present study we can make the following conclusions:

1. Large values of buoyancy parameter $(\lambda)$ can be used to control the temperature and concentration boundary layers.

2. Suction $(F w)$ stabilizes the boundary layer growth.

3. There exists a high influence of Prandti number $(\mathrm{Pr})$ on velocity, temperature and concentration profiles.

4. Heat source $(Q)$ cause reduction in the concentration distribution in the concentration boundary layer.

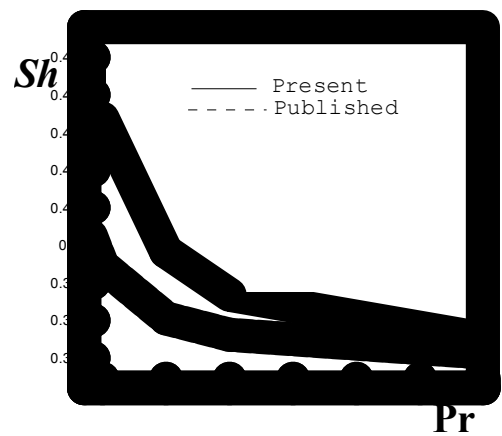

5. Existence of heavier species Schmidt number $(S c)$ decrease the fluid velocity, heat transfer and concentration boundary layer.

\section{Nomenclature}

C Species concentration in the flow field

$C_{w} \quad$ Species concentration at the wall

$C_{\mathrm{or}} \quad$ Species concentration in the free stream

$C_{f} \quad$ Skin friction coefficient

$C_{p} \quad$ Specific heat at constant pressure

$D_{m} \quad$ Coefficient of mass diffusivity

$f$ Dimensionless stream function 
Fw Suction parameter

$\mathrm{Gr}$ Grashof number

9 Acceleration due to gravity

M Magnetic field parameter

$N \quad$ Radiation parameter

$q . \quad$ Radiative heat flux (Rosseland approximation)

Pr Prandtl number

$Q \quad$ Heat source parameter

Re Reynolds number

5c Schmidt number

sh Sherwood number

$\tau_{W}$ Temperature at the wall

$T_{\infty} \quad$ Free stream temperature

$u \quad$ Velocity along $\mathrm{x}$-axis

$v \quad$ Velocity along $\mathrm{y}$-axis

$v_{w} \quad$ Suction velocity

$x \quad$ Coordinate along the plate

$y \quad$ Coordinate normal the plate

\section{Greek Symbols}

$\begin{array}{ll}\alpha & \text { Thermal diffusivity } \\ \theta & \text { Coefficient of volume expansion } \\ \theta & \text { Dimensionless temperature } \\ \rho & \text { Density of the fluid } \\ \mu & \text { Coefficient of viscosity } \\ \sigma & \text { Electric conductivity } \\ \sigma_{1} & \text { Stefan-Boltzmann constant } \\ \varphi & \text { Dimensionless concentration } \\ \eta & \text { Similarity variable } \\ \lambda & \text { Buoyancy parameter } \\ v & \text { Kinematic viscosity } \\ \tau & \text { Shear stress } \\ \psi & \text { Stream function } \\ \kappa & \text { Thermal conductivity } \\ \kappa_{1} & \text { Mean absorption coefficient }\end{array}$

1. Sakiadis B. C., "Boundary layer behavior on continuous solid surfaces: I The Boundary layer equations for two-dimensional and axi-symmetric flow", AIChE J. 7(1), 26-28 (1961)

2. Sakiadis B. C., "Boundary layer behavior on continuous solid surface: II The boundary layer on a continuous flat surface", AIChE J. 7(1), 221-225 (1961)

3. Erickson L. E., L.T. Fan and V.G. Fox, "Heat and mass transfer on a moving continuous flat plate with suction and injection", Ind. Eng. Chem. Fundam:, 5, 19-25 (1966)

4. Tsouo F. K., F.M. Sparrow and R.J. Goldstein, "Flow and heat transfer in the boundary layer in continuous moving surface", Int. J. Heat Mass Transfer, 10, 219-235 (1967)

5. Crane L. J., "Flow past a Stretching plate", ZAMP, 21 (4), 645-667 (1970)

6. Vajravelu K. and A. Hadjiricaloou , "Convective heat transfer in anelectrically conducting fluid at a stretching surface with uniform free stream", Int. J. Eng. Sci., 35,1237-1244 (1997)

7. Gebhart B., "Effects of viscous dissipation in Natural convection", J. Fluid Mech., 14, 225-232 (1962)

8. Khaleque Tania S. and M.A. Samad, "Effects of Radiation, heat generation and viscous dissipation on MHD free convection flow along a stretching sheet", Res. J. Appl. Sci. Eng. Technology, 2(4), 368-377 (2010)

9. Anderson H. I., O.R. Hansen and B. Holmedal, "Diffusion of a chemical reaction species from a stretching sheet", Int. J. Heat and Mass Transfer, 37(4), 659-664 (1994)

10. Anjali Devi S. P. and R. Kandasamy, "Effects of chemical reaction, heat and mass transfer on laminar flow along a semi infinite horizontal plate", Warme-und Stoffubertragung Zeit Schrift, 35(6), 465-467 (1999)

11. Nachtsheim P. R. and P. Swigert, "Satisfaction of the asymptotic boundary conditions in numerical solution of the system of non-linear equations of boundary layer type", $N A S A$ TND-3004 (1965).

12. Samad M. A. and M. Mohebujjaman, "MHD Heat and Mass Transfer Free Convection Flow along a Vertical Stretching Sheet in Presence of Magnetic Field with Heat Generation", Res. J. Appl. Sci. Eng. Technology, 1(3), 98-106 (2009). 
Dhaka Univ. J. Sci. 61(1): 27-34, 2013 (January) 